\title{
Clinical efficacy of perampanel for partial-onset and primary generalized tonic-clonic seizures
}

\author{
This article was published in the following Dove Press journal: \\ Neuropsychiatric Disease and Treatment \\ 17 May 2016 \\ Number of times this article has been viewed
}

\section{Frank MC Besag ${ }^{1-3}$ \\ Philip N Patsalos ${ }^{4,5}$}

'East London Foundation NHS Trust, ${ }^{2}$ Institute of Psychiatry, Psychology and Neuroscience, ${ }^{3} \mathrm{UCL}$ School of Pharmacy, ${ }^{4}$ Department of Clinical and Experimental Epilepsy, UCL Institute of Neurology, NIHR University College London Hospitals Biomedical Research Centre, London, ${ }^{5}$ Epilepsy Society, Chalfont Centre for Epilepsy, Chalfont St Peter, UK
Correspondence: Philip N Patsalos Department of Clinical and Experimental Epilepsy, UCL Institute of Neurology, Queen Square, London WCIN 3BG, UK Tel +44 I494 60 I355

Fax +44 I494 $875 I 76$

Email p.patsalos@ucl.ac.uk
Background and purpose: Perampanel, a selective noncompetitive antagonist at the $\alpha$-amino-3-hydroxy-5-methyl-4-isoxazolepropionic acid (AMPA) receptor, is highly effective in a wide range of experimental models. Although initially licensed as adjunctive therapy for partial seizures with or without secondary generalization in patients aged 12 years or older, the US Food and Drug Administration has recently approved its use in the treatment of primary generalized tonic-clonic seizures (PGTCS). This paper reviews the pharmacokinetics, efficacy, and tolerability of perampanel as an antiepileptic drug.

Results: After oral ingestion, perampanel is rapidly absorbed ( $T_{\max }, 0.5-2.5$ hours), has a bioavailability of $\sim 100 \%$, and is highly protein bound ( $95 \%)$ in plasma. It undergoes extensive (>90\%) hepatic metabolism, primarily via cytochrome P450 3A4 (CYP3A4), with a half-life of 48 hours. Carbamazepine and other antiepileptic drugs can enhance its metabolism via induction of CYP3A4. Efficacy of perampanel in focal seizures has been extensively evaluated in Phase II and randomized, placebo-controlled Phase III trials. The efficacy in PGTCS has been reported in one class I study. In the treatment of focal seizures, perampanel showed significant dosedependent median seizure reductions: $4 \mathrm{mg} / \mathrm{d}, 23 \%$; $8 \mathrm{mg} / \mathrm{d}, 26 \%-31 \% ; 12 \mathrm{mg} / \mathrm{d}, 18 \%-35 \%$; and placebo, $10 \%-21 \%$. The $50 \%$ responder rates were $15 \%-26 \%, 29 \%, 33 \%-38 \%$, and $34 \%-36 \%$ for placebo, $4 \mathrm{mg} / \mathrm{d}, 8 \mathrm{mg} / \mathrm{d}$, and $12 \mathrm{mg} / \mathrm{d}$ perampanel, respectively. Freedom from seizures was recorded in $0 \%-1.7 \%$ of the placebo group, $1.9 \%$ of the 2 mg group, $2.6 \%-4.4 \%$ of the $8 \mathrm{mg}$ group, and $2.6 \%-6.5 \%$ of the $12 \mathrm{mg}$ group. For PGTCS, the median seizure reduction was $76.5 \%$ for perampanel and $38.4 \%$ for placebo. The $50 \%$ responder rate was $64.2 \%$ for perampanel and $39.5 \%$ for placebo. Seizure freedom during maintenance phase was $30.9 \%$ for perampanel and $12.3 \%$ for placebo. Adverse effects included dose-dependent increases in the frequency of dizziness, somnolence, fatigue, irritability, falls, and probably nausea.

Conclusion: Perampanel is effective in treating both partial-onset seizures and PGTCS.

Keywords: perampanel, new antiepileptic drug, epilepsy, primary generalized seizures, pharmacokinetics

\section{Introduction}

The number of antiepileptic drugs (AEDs) with specific efficacy in primary generalized tonic-clonic seizures (PGTCS) is limited. The majority of AEDs have been developed for treating focal seizures, with or without secondary generalization. Although some of these, eg, carbamazepine, are also licensed for treating generalized tonic-clonic seizures, it is possible that the seizures that respond to such AEDs are actually of focal onset. An indication that this might be the case is that seizure control tends to deteriorate when carbamazepine is prescribed for the classic generalized epilepsy, namely juvenile myoclonic epilepsy. The AEDs that fulfill the criterion of treating tonic-clonic seizures in juvenile myoclonic epilepsy include sodium valproate, lamotrigine, and 
levetiracetam. The benzodiazepine drugs may also be effective but are not usually prescribed because of problems with tolerance and adverse effects. There is a need for additional AEDs that treat PGTCS. In this paper, we review the mode of action, pharmacokinetics, efficacy, and adverse effects of the new AED perampanel in patients with focal seizures and in patients with PGTCS.

\section{Perampanel mode of action In vitro studies}

In vitro studies have shown that perampanel is a selective, noncompetitive antagonist at the $\alpha$-amino-3-hydroxy-5-methyl4-isoxazolepropionic acid (AMPA) receptor. ${ }^{1,2}$ In studies in rat cortical neurons, perampanel has been shown to potently inhibit AMPA-evoked calcium currents $\left(\mathrm{IC}_{50}=93 \mathrm{nM}\right.$ [concentration of inhibitor where response is reduced by $50 \%$ ]) compared with GYKI52466, which is a noncompetitive AMPA receptor antagonist $\left(\mathrm{IC}_{50}=12.5 \mu \mathrm{M}\right) .{ }^{3}$ Furthermore, rat hippocampal slice studies show that perampanel is selectively active on AMPA receptors over other receptors. ${ }^{4}$ Indeed, perampanel has no effect on responses mediated by $N$-methylD-aspartate (NMDA) receptors or kainate receptors. ${ }^{4}$

\section{Efficacy of perampanel in animal seizure models}

Perampanel is effective in various seizure models, including the mouse tonic-clonic generalized seizure model, the audiogenic and maximal electric shock-induced seizure tests, and the pentylenetetrazole-induced seizure model. Perampanel also inhibits $6 \mathrm{~Hz}$ electroshock-induced seizures but is inactive in genetic absence epilepsy rats from Strasbourg. ${ }^{3}$

\section{Perampanel pharmacokinetic characteristics}

The therapeutic effect of a drug in terms of dosing frequency is critically determined by its pharmacokinetic characteristics, which influence the recommended rate of dose titration, the time course of achieving an optimum therapeutic response, and patient compliance. Furthermore, because all new AEDs are licensed, at least initially, as adjunctive therapy, knowledge of their propensity to interact with concomitant drugs is important. ${ }^{5,6}$ Successful therapy or failure will be determined not only by efficacy but also by these considerations.

\section{Absorption and distribution}

After oral ingestion, perampanel is rapidly absorbed (time to maximum concentration $\left[T_{\max }\right], 0.5-2.5$ hours), is not subject to any significant first-pass metabolism, and has a bioavailability of almost $100 \%$. It is highly protein bound ( $95 \%)$ in plasma, primarily to albumin, and has a volume of distribution of $1.1 \mathrm{~L} / \mathrm{kg}$ in adults. Co-ingestion with food delays the rate of absorption (maximum concentration $\left[C_{\max }\right]$ values are decreased by $40 \% ; T_{\max }$ is delayed by 2 hours), but it does not affect the extent of absorption. In healthy individuals and patients with epilepsy, perampanel demonstrates linear pharmacokinetics over the dose range of $2-12 \mathrm{mg} / \mathrm{d}$ and the time to steady state is $10-19$ days. $^{7}$

\section{Metabolism}

Perampanel undergoes extensive hepatic metabolism $(>90 \%)$ to form 13 major metabolites of hydroxylated perampanel and various glucuronide conjugates, none of which are pharmacologically active. The isoenzyme CYP3A4 is primarily considered to be involved in the metabolism of perampanel, although CYP3A5 may also contribute. ${ }^{7}$

\section{Elimination}

The calculated mean plasma half-life of single-dose perampanel in healthy adult volunteers is 48 hours. In the presence of enzyme-inducing AEDs (eg, carbamazepine), perampanel half-life values can decrease to $\sim 25$ hours. Only $\sim 2 \%$ of an administered perampanel dose is excreted as unchanged perampanel in urine, while $70 \%$ of a dose is excreted in feces. ${ }^{7}$

\section{Drug interaction profile}

Perampanel is not expected to cause pharmacokinetic interactions, because, at clinically relevant concentrations, in vitro, it is neither a potent inhibitor nor an inducer of cytochrome P450 (CYP) isoenzymes or uridine diphosphate glucuronosyltransferases. However, perampanel will be the target of drug interactions, because CYP3A4, the principal isoenzyme responsible for its metabolism, is readily induced and inhibited. ${ }^{7}$ Concomitant AEDs that are CYP3A4 inducers (eg, carbamazepine, oxcarbazepine, phenytoin, and topiramate) will increase perampanel clearance and decrease perampanel plasma concentrations. Conversely, strong inhibitors of CYP3A4 (eg, ketoconazole) will decrease perampanel clearance and increase perampanel plasma concentrations. Although perampanel does not interact with combined oral contraceptives (when dosed at $\leq 8 \mathrm{mg} / \mathrm{d}$ ), perampanel at $12 \mathrm{mg}$ decreases the concentration of levonorgestrel and could possibly compromise contraceptive control. 


\section{Efficacy of perampanel in human studies \\ Partial seizures with or without secondary generalization}

The efficacy of perampanel has been extensively evaluated in various Phase II, ${ }^{8}$ randomized placebo-controlled Phase III, ${ }^{9-11}$ and open-label extension trials, ${ }^{12}$ as well as in postmarketing observational studies. ${ }^{13,14}$ Furthermore, pooled analysis of the five Phase II/Phase III trials has been undertaken. ${ }^{15,16}$ Patients recruited were $\geq 12$ years of age, had refractory partial seizures with or without secondary generalization, and were on 1-3 additional AEDs.

The Phase III studies showed dose-dependent, statistically significant median seizure reductions: $4 \mathrm{mg} / \mathrm{d}, 23 \%$; $8 \mathrm{mg} / \mathrm{d}, 26 \%-31 \% ; 12 \mathrm{mg} / \mathrm{d}, 18 \%-35 \%$; and placebo, $10 \%-21 \%{ }^{9-11}$ Perampanel $2 \mathrm{mg} / \mathrm{d}$ was not associated with any significant seizure reduction. The $50 \%$ responder rates were $15 \%-26 \%, 29 \%, 33 \%-38 \%$, and $34 \%-36 \%$ for placebo, 4, 8 , and $12 \mathrm{mg} / \mathrm{d}$ perampanel, respectively. Freedom from seizures was recorded in $0 \%-1.7 \%$ of the placebo group, $1.9 \%$ of the $2 \mathrm{mg}$ group, $2.6 \%-4.4 \%$ of the $8 \mathrm{mg}$ group, and $2.6 \%-6.5 \%$ of the $12 \mathrm{mg}$ group. Pooled analysis of these data confirmed the significant efficacy of perampanel, with median seizure reduction rates of $23.3 \%, 28.8 \%$, and $27.2 \%$ for 4,8 , and $12 \mathrm{mg} / \mathrm{d}$, respectively; the placebo effect was $12.8 \% .^{15,16}$ Perampanel at a dose of $12 \mathrm{mg} / \mathrm{d}$ did result in improved efficacy in some patients. ${ }^{17}$ Analysis of the pooled data suggested that perampanel had efficacy against secondarily generalized tonic-clonic seizures. ${ }^{18}$

Since perampanel was licensed for clinical use, its efficacy has been further characterized from reports describing its use in everyday clinical practice ${ }^{13,14}$ and also from an openlabel extension study of the core Phase III studies. ${ }^{12}$ The study by Steinhoff et a ${ }^{13}$ included 74 patients ( 43 females) aged 15-71 years (mean age: 38.4 years) who were followed up for 6-7 months. Perampanel was administered in an initial dose of $2 \mathrm{mg} / \mathrm{d}$ for 14 days followed up by a $4 \mathrm{mg} / \mathrm{d}$ dose for a further 4 weeks. Subsequently, the perampanel dose was titrated to a maximum individual tolerated dose; one patient achieved a dose of $14 \mathrm{mg} / \mathrm{d}$. Comparing the last 3 months of observation to baseline revealed that 34 patients $(46 \%)$ responded to treatment with at least a $50 \%$ reduction in seizure frequency. Of the 34 patients, ten became seizure free. Of the 43 patients co-prescribed enzyme-inducing AEDs, 18 patients (42\%) responded to treatment, compared with $48 \%$ (15 patients) who were responders but were not co-prescribed enzyme-inducing
AEDs. A subsequent multicenter study, which used the same study design but included 281 patients (152 females) with a mean age of 39 years (range: $12-84$ years), reported similar findings. ${ }^{14}$ At the end of a 6-month follow-up, retention was $60 \% ; 50 \%$ of patients had at least a $50 \%$ reduction in seizure frequency.

The open-label extension study described by Montouris et al ${ }^{12}$ included 1,281 patients ( $50 \%$ females) with a median age of 33 years (range: 12-76 years). During the first 16 weeks, patients who were already taking perampanel during one of the Phase III clinical trials continued to do so blinded, but if they were not already receiving $12 \mathrm{mg} / \mathrm{d}$ the dose was titrated to $12 \mathrm{mg} / \mathrm{d}$ or up to the maximally tolerated individual dose. For patients who had initially been randomized to placebo in the Phase III studies, perampanel was titrated during a 16-week period. After an initial dose of $2 \mathrm{mg} / \mathrm{d}$, perampanel was increased by $2 \mathrm{mg} / \mathrm{d}$ every 14 days up to a target dose of $10-12 \mathrm{mg} / \mathrm{d}$. After a 52-week follow-up, mean seizure frequency decreased from baseline by $55 \%$ and $53.9 \%$ in the initial placebo and perampanel groups, respectively. Furthermore, the $50 \%$ responder rates were $55 \%$ and $52.8 \%$, respectively.

Shah et a $\mathrm{l}^{17}$ recently published a pooled, retrospective, case-note data study of 310 adult patients (mean age, 40.9 years; range: $18-75$ years; $50 \%$ females; $27.7 \%$ intellectual disability). Only eight patients were said to have idiopathic generalized epilepsy. A total of 161 patients were treated for 6 months or longer. The 50\% responder rates in the 3 months before the last follow-up were $57.5 \%$ for tonic-clonic seizures, $74 \%$ for complex partial (dyscognitive) seizures, and $14 \%$ for simple partial seizures. Adverse events were recorded in 209 patients (67.4\%), and, of these, $67 \%$ withdrew from the perampanel because of adverse events. The most common adverse events were sedation $23.8 \%$, behavioral $/$ mood disturbance $22.6 \%$, dizziness $13.5 \%$, unsteadiness $11.3 \%$, and increase in seizure frequency $7.1 \%$. There was a significant increase in the prevalence of dizziness with fast titration, increasing the dose by $>2 \mathrm{mg} / 2 \mathrm{wks}$ $(P=0.025)$. The authors drew attention to the high rate of irritability/aggression: $18.1 \%$; in addition, $7.7 \%$ had mood change/anxiety. The rate of behavioral disturbance was much higher than that of $4.9 \%$ (aggression/irritability) reported by Steinhoff et al in the German/Austrian study, ${ }^{14}$ or the $1.6 \%$ (aggression) reported in the pooled analysis of the Phase III studies. ${ }^{16}$ The retention rate fell from $86 \%$ at 3 months to $27 \%$ at 18 months. The efficacy and adverse event results for the subgroup with intellectual disability were similar to those 
for the whole group, but simple partial seizures were not assessed because of difficulty in recording these accurately in this group.

The first pediatric use of perampanel has been reported in a series of 58 patients (32 females) with a mean age of $10.5 \pm 4.2$ years (range: $2-17$ years). ${ }^{19}$ Although perampanel was usually started at $2 \mathrm{mg} / \mathrm{d}$ and titrated by $2 \mathrm{mg} / \mathrm{wk}$ or $2 \mathrm{mg} / 2 \mathrm{wks}, 1 \mathrm{mg}$ dose steps and slower titration rates were used in children $\leq 12$ years. After 3 months of perampanel therapy, 18 patients $(31 \%)$ exhibited a $50 \%$ response rate and five patients $(9 \%)$ became seizure free.

An audit of perampanel use in 47 patients ( 24 females; median age, 31 years; range: 18-61) attending a regional epilepsy service revealed a $50 \%$ responder rate of $28 \%$, with 21 patients $(45 \%)$ withdrawing from perampanel treatment. ${ }^{20}$ The dosing strategy was that typically employed for perampanel, namely $2 \mathrm{mg} / \mathrm{d}$ initially, increased by $2 \mathrm{mg} / \mathrm{d}$ every 14 days.

\section{Primary generalized seizures}

There is a single published study of the efficacy and tolerability of perampanel in primary generalized epilepsy. ${ }^{21}$ This was an international, parallel-group, multicentre, doubleblind study in patients 12 years of age or older. It provided class I evidence of efficacy compared with placebo. A total of 164 patients were randomized, of whom 162 were included in the full analysis: 81 treated with placebo and 81 with perampanel.

The investigators paid particular attention to the correct diagnosis of established "idiopathic" generalized epilepsy; a large proportion of the patients initially submitted were excluded on the basis of this reassessment. The "randomization phase" included titration over weeks $1-4$, maintenance over weeks 5-17, and follow-up (only for patients not entering an extension phase) over weeks 18-21. The active perampanel and the placebo were packaged and labeled so as to be indistinguishable. The initial perampanel dose was $2 \mathrm{mg}$. The dose was increased in increments of $2 \mathrm{mg}$ to a target dose of $8 \mathrm{mg}$ or to the highest tolerated dose, whichever was lower. The final dose achieved during this phase was continued in the maintenance phase. Alteration of the dose was "not recommended" during the maintenance phase, but patients with inadequate seizure control could have the dose increased by one $2 \mathrm{mg}$ increment (but not $>8 \mathrm{mg}$ ) or patients with intolerable adverse events could have a dose reduction by one $2 \mathrm{mg}$ increment. Seizure counts were recorded in patient diaries. The primary efficacy endpoint was the percentage change in PGTCS frequency per 28 days, including both the titration and maintenance phases, compared with the baseline. The secondary efficacy endpoint was the percentage of patients with a reduction in PGTCS of $50 \%$ or more (the responder rate). "Exploratory" efficacy endpoints included the rate of freedom from PGTCS, the rate of freedom from all seizures during the maintenance phase, and the investigator-assessed Clinical Global Impression of Change, rated on a scale from 1 (very much improved) to 7 (very much worse) - last observation was carried forward throughout the 4 weeks before week 12. Biochemical, hematological, and urinalysis tests were performed. Vital signs and treatmentemergent adverse events were recorded. The efficacy results were as follows. Median percentage change in PGTCS per 28 days: perampanel $-76.5 \%$; placebo $-38.4 \% ; P<0.0001$. PGTCS responder rate: perampanel $64.2 \%$; placebo $39.5 \%$; $P=0.0019$. Seizure freedom during maintenance phase: perampanel $30.9 \%$; placebo $12.3 \%$. The tolerability results were as follows. In each case, the treatment-emergent adverse events for perampanel were stated and the figure for placebo appears in parenthesis: any treatment-emergent adverse event $82.7 \%$ (72.0\%), dizziness 32.1\% (6.1\%), fatigue $14.8 \%$ (6.1\%), headache $12.3 \%$ (9.8\%), somnolence $11.1 \%$ (3.7\%), irritability $11.1 \%(2.4 \%)$, nasopharyngitis $8.6 \%$ (8.5\%), vertigo $8.6 \%(2.4 \%)$, vomiting $8.6 \%$ (2.4\%), weight increased $7.4 \%(3.7 \%)$, and nausea $6.2 \%(4.9 \%)$. The authors concluded that the most frequent treatment-emergent adverse events with perampanel were dizziness (32.1\%) and fatigue (14.8\%). However, it is difficult to draw any meaningful conclusions with regard to adverse effects from these relatively small numbers. Furthermore, no definitive conclusions can be drawn with regard to safety from the relatively small numbers treated in this trial or, indeed, from the total number treated in all the published clinical trials so far. Much larger numbers would be required. The authors concluded that their study provided class I evidence that adjunctive perampanel decreases PGTCS frequency compared with placebo in drug-resistant patients.

There are two case reports on the efficacy of perampanel in PGTCS in progressive myoclonus epilepsy. Schorlemmer et $\mathrm{al}^{22}$ described a case of a 21 -year-old woman with progressive myoclonus epilepsy (Lafora body disease), confirmed by the identification of a mutation in the EPM $2 A$ gene, who had a sustained remission of myoclonus and PGTCS with perampanel $8 \mathrm{mg} / \mathrm{d}$. When the dose was reduced to $6 \mathrm{mg} / \mathrm{d}$, the seizures recurred, but when the dose was increased again to $10 \mathrm{mg}$ the seizures stopped and she regained the ability to walk with the aid of a walker. Dirani et $\mathrm{al}^{23}$ described a 15 -year-old girl with Lafora body disease and refractory epilepsy. Perampanel was titrated rapidly to $10 \mathrm{mg} / \mathrm{d}$ over a 12-day period. Approximately 2 weeks after the perampanel was started, there was a marked improvement in both the myoclonus and the PGTCS frequency. 
There was also a marked improvement in the activities of daily living. Her speech and motor skills improved, and her parents said that her memory also improved. She was able to eat and drink independently. She was discharged taking perampanel 8 and $12 \mathrm{mg}$ daily on alternate days.

\section{Adverse effects}

Distinguishing adverse effects from adverse events in clinical trials is subject to a number of confounding factors. Although the simplest approach to allow for these confounding factors is to compare the rate of adverse events in the perampaneltreated group with the placebo-treated group, such grouped data do not allow for variables such as drug dosage, concomitant medication, and individual patient characteristics. Different studies may also use different methods of assessing adverse events. If allowance is made for such factors, there appears to be no obvious reason why the adverse effects of perampanel used for treating partial-onset seizures should be any different from the adverse effects when it is used for treating primary generalized seizures. Using pooled data has the major advantage of providing large numbers; for this reason, the pooled data from studies in partial-onset seizures have been compared with the data from the single study on PGTCS. A large pooled dataset on perampanel for the treatment of partial-onset seizures is available from Montouris et al. ${ }^{12}$ In their analysis, 838 patients received perampanel during the core studies and continued with perampanel treatment during the extension studies. The most common adverse events (greater than 10\%) were as follows: dizziness $47.5 \%$, somnolence $22.4 \%$, headache $22 \%$, fatigue $14.1 \%$, weight increased $10.5 \%$, nasopharyngitis $10.3 \%$. However, these figures do not control for placebo effects. The data from the study by French et $\mathrm{al}^{21}$ on PGTCS do allow a comparison with placebo. The most common adverse events (again, greater than $10 \%$ ) compared with the placebo rates (in parenthesis) were as follows: dizziness $32.1 \%$ (6.1\%), fatigue $14.8 \%$ (6.1\%), headache $12.3 \%(9.8 \%)$, somnolence $11.1 \%(3.7 \%)$, irritability $11.1 \%(2.4 \%)$. Taken together, and allowing for placebo effects, it would appear from these data that dizziness, fatigue, somnolence, and irritability are likely to be not only adverse events but adverse effects of perampanel, although further information from larger numbers of patients will be required before definitive statements can be made.

Another valuable way of providing information on adverse effects is to document the effect of dose on the rate of adverse events. If there is a clear relationship between the rate of an adverse event and the dose of the drug, it is highly likely that this is an adverse effect of the drug. Pooled data from three studies on partial-onset seizures, using

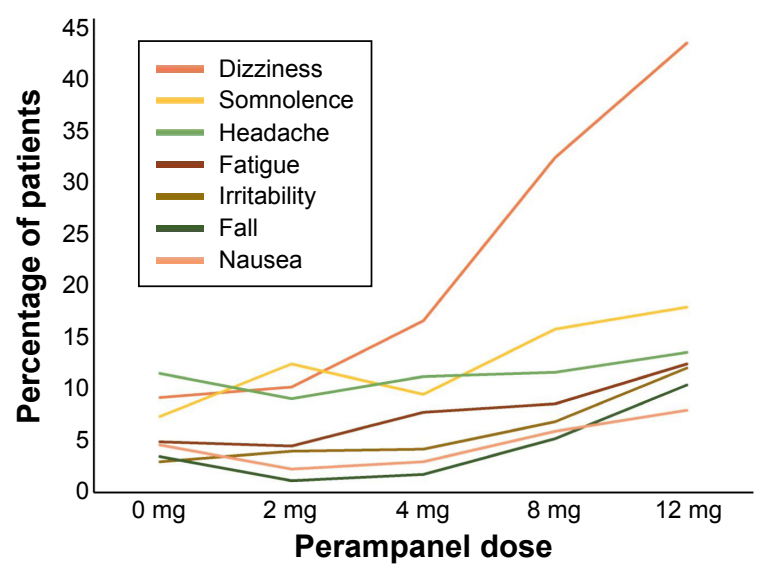

Figure I Frequency of treatment-emergent adverse events for perampanel plotted against dose.

Note: The placebo results are plotted as $0 \mathrm{mg}$.

information extracted from the tabulated summary provided by Rugg-Gunn, ${ }^{24}$ are shown in Figure 1.

This graph shows a clear relationship between dose and dizziness, somnolence, fatigue, irritability, falls, and probably nausea. The dose-relationship data highlight another issue which, although not surprising, is of clinical importance, namely that the rate of adverse effects is likely to be low if the perampanel dose is kept to a minimum. A recent analysis by Brodie et al (unpublished data, 2016) has indicated that the behavioral effects of perampanel, particularly aggression, appear to be dose related and are much less likely to occur at lower doses.

\section{Conclusion}

Although there is only one double-blind randomized controlled trial on the efficacy of perampanel in PGTCS, this appears to be a well-conducted study that provides unequivocal, highly statistically significant, class I evidence for efficacy. The tolerability of perampanel appears to be broadly similar to other AEDs; the most frequent adverse effects were dizziness and fatigue, although it should be noted that there have been reports of irritability and behavioral disturbance with higher doses than those used in this study. The total numbers of patients in reported trials remain too small to allow definitive statements to be made with regard to safety. On the basis of the current evidence, perampanel would appear to be a valuable addition to the available AEDs for the treatment of partial-onset seizures and to the limited choice available for the treatment of PGTCS. However, until it has been much more widely used in clinical practice, it will not be possible to determine what its final role in the treatment of patients with epilepsy will be. 


\section{Acknowledgments}

The contribution of FMCB to this work was not carried out in relation to any specific appointment but will be listed as part of the work carried out by this author in the appointments listed at the beginning of this paper, and the contribution of PNP to this work was undertaken at the University College Hospitals (UCLH)/University College London (UCL) Comprehensive Bio-Medical Research Centre, which received a proportion of funding from the Department of Health's NIHR Biomedical Research Centres funding scheme.

\section{Disclosure}

FMCB has no conflict of interest to declare with regard to this manuscript. He has attended conferences and has recently taken part in writing a review paper funded with support from unrestricted educational grants from Eisai, the pharmaceutical company responsible for perampanel. He has received no personal monies from this company. PNP reports no conflicts of interest in this work.

\section{References}

1. Rogawski MA. Revisiting AMPA receptors as an antiepileptic drug target. Epilepsy Curr. 2011;11(2):56-63.

2. Rogawski MA, Hanada T. Preclinical pharmacology of perampanel, a selective non-competitive AMPA receptor antagonist. Acta Neurol Scand. 2013;127:19-24.

3. Hanada T, Hashizume Y, Tokuhara N, et al. Perampanel: a novel, orally active, noncompetitive AMPA-receptor antagonist that reduces seizure activity in rodent models of epilepsy. Epilepsia. 2011;52(7): 1331-1340.

4. Ceolin L, Bortolotto ZA, Bannister N, Collingridge GL, Lodge D, Volianskis A. A novel anti-epileptic agent, perampanel, selectively inhibits AMPA receptor-mediated synaptic transmission in the hippocampus. Neurochem Int. 2012;61(4):517-522.

5. Patsalos PN. Drug interactions with the Newer Antiepileptic Drugs (AEDs) - part 1: pharmacokinetic and pharmacodynamic interactions between AEDs. Clin Pharmacokinet. 2013;52(11):927-966.

6. Patsalos PN. Drug Interactions with the Newer Antiepileptic Drugs (AEDs) - part 2: pharmacokinetic and pharmacodynamic interactions between AEDs and drugs used to treat non-epilepsy disorders. Clin Pharmacokinet. 2013;52(12):1045-1061.

7. Patsalos PN. The clinical pharmacology profile of the new antiepileptic drug perampanel: a novel noncompetitive AMPA receptor antagonist. Epilepsia. 2014;56(1):12-27.

8. Krauss GL, Bar M, Biton V, et al. Tolerability and safety of perampanel: two randomized dose-escalation studies. Acta Neurol Scand. 2012; 125(1):8-15.

Neuropsychiatric Disease and Treatment

\section{Publish your work in this journal}

Neuropsychiatric Disease and Treatment is an international, peerreviewed journal of clinical therapeutics and pharmacology focusing on concise rapid reporting of clinical or pre-clinical studies on a range of neuropsychiatric and neurological disorders. This journal is indexed on PubMed Central, the 'PsycINFO' database and CAS,
9. Krauss GL, Serratosa JM, Villanueva V, et al. Randomized phase III study 306: adjunctive perampanel for refractory partial-onset seizures. Neurology. 2012;78(18):1408-1415.

10. French JA, Krauss GL, Biton V, et al. Adjunctive perampanel for refractory partial-onset seizures: randomized phase III study 304. Neurology. 2012;79(6):589-596.

11. French JA, Krauss GL, Steinhoff BJ, et al. Evaluation of adjunctive perampanel in patients with refractory partial-onset seizures: results of randomized global phase III study 305. Epilepsia. 2013;54(1):117-125.

12. Montouris G, Yang H, Williams B, Zhou S, Laurenza A, Fain R. Efficacy and safety of perampanel in patients with drug-resistant partial seizures after conversion from double-blind placebo to open-label perampanel. Epilepsy Res. 2015;114:131-140.

13. Steinhoff BJ, Bacher M, Bast T, et al. First clinical experiences with perampanel-the Kork experience in 74 patients. Epilepsia. 2014;55: 16-18.

14. Steinhoff BJ, Hamer H, Trinka E, et al. A multicenter survey of clinical experiences with perampanel in real life in Germany and Austria. Epilepsy Res. 2014;108(5):986-988.

15. Hsu WW, Sing CW, He Y, Worsley AJ, Wong IC, Chan EW. Systematic review and meta-analysis of the efficacy and safety of perampanel in the treatment of partial-onset epilepsy. CNS Drugs. 2013; 27(10):817-827.

16. Steinhoff BJ, Ben-Menachem E, Ryvlin P, et al. Efficacy and safety of adjunctive perampanel for the treatment of refractory partial seizures: a pooled analysis of three phase III studies. Epilepsia. 2013;54(8): 1481-1489.

17. Shah E, Reuber M, Goulding P, Flynn C, Delanty N, Kemp S. Clinical experience with adjunctive perampanel in adult patients with uncontrolled epilepsy: a UK and Ireland multicentre study. Seizure. 2016; 34:1-5.

18. Kramer LD, Satlin A, Krauss GL, et al. Perampanel for adjunctive treatment of partial-onset seizures: a pooled dose-response analysis of phase III studies. Epilepsia. 2014;55(3):423-431.

19. Biro A, Stephani U, Tarallo T, et al. Effectiveness and tolerability of perampanel in children and adolescents with refractory epilepsies: first experiences. Neuropediatrics. 2015;46(2):110-115.

20. Coyle H, Clough P, Cooper P, Mohanraj R. Clinical experience with perampanel: focus on psychiatric adverse effects. Epilepsy Behav. 2014; 41:193-196.

21. French JA, Krauss GL, Wechsler RT, et al. Perampanel for tonic-clonic seizures in idiopathic generalized epilepsy: a randomized trial. Neurology. 2015;85(11):950-957.

22. Schorlemmer K, Bauer S, Belke M, et al. Sustained seizure remission on perampanel in progressive myoclonic epilepsy (Lafora disease). Epilepsy Behav Case Rep. 2013;1:118-121.

23. Dirani M, Nasreddine W, Abdulla F, Beydoun A. Seizure control and improvement of neurological dysfunction in Lafora disease with perampanel. Epilepsy Behav Case Rep. 2014;2:164-166.

24. Rugg-Gunn F. Adverse effects and safety profile of perampanel: a review of pooled data. Epilepsia. 2014;55(suppl 1):13-15. and is the official journal of The International Neuropsychiatric Association (INA). The manuscript management system is completely online and includes a very quick and fair peer-review system, which is all easy to use. Visit http://www.dovepress.com/testimonials.php to read real quotes from published authors. 